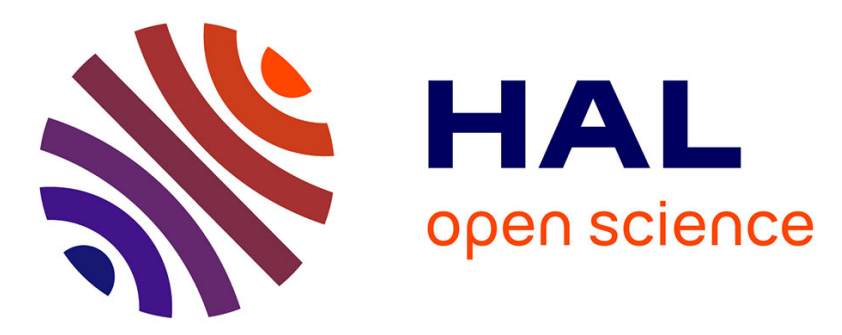

\title{
Estimation of the flow of particles within a partition of the image domain in fluorescence video-microscopy
} Thierry Pécot, Jérôme Boulanger, Charles Kervrann, Patrick Bouthemy, Jean Salamero

\section{- To cite this version:}

Thierry Pécot, Jérôme Boulanger, Charles Kervrann, Patrick Bouthemy, Jean Salamero. Estimation of the flow of particles within a partition of the image domain in fluorescence video-microscopy. ISBI 11th IEEE International Symposium On Biomedical Imaging: from nano to macro - 2014, Apr 2014, Beijing, China. hal-00936063

\section{HAL Id: hal-00936063 https://hal.inria.fr/hal-00936063}

Submitted on 24 Jan 2014

HAL is a multi-disciplinary open access archive for the deposit and dissemination of scientific research documents, whether they are published or not. The documents may come from teaching and research institutions in France or abroad, or from public or private research centers.
L'archive ouverte pluridisciplinaire HAL, est destinée au dépôt et à la diffusion de documents scientifiques de niveau recherche, publiés ou non, émanant des établissements d'enseignement et de recherche français ou étrangers, des laboratoires publics ou privés. 


\title{
Estimation of the flow of particles within a partition of the image domain in fluorescence video-microscopy
}

\author{
Thierry Pécot*, Jérôme Boulanger ${ }^{\dagger}$, Charles Kervrann*, Patrick Bouthemy*, Jean Salamero ${ }^{\dagger}$ \\ * Inria, Centre Rennes - Bretagne Atlantique, Campus Universitaire de Beaulieu, 35042 Rennes Cedex, France \\ $\dagger$ UMR 144 CNRS / Institut Curie, 12 rue Lhomond, 75005 Paris, France
}

\begin{abstract}
Automatic analysis of the dynamic content in fluorescence video-microscopy is crucial for understanding molecular mechanisms involved in cell functions. In this paper, we propose an original approach for analyzing particle trafficking in these sequences. Instead of individually tracking every particle, we estimate the particle flows between predefined regions. This approach allows us to process image sequences with a high number of particles and a low frame rate. We investigate several ways to estimate the particle flow at the cellular level and evaluate their performance in synthetic and real image sequences.
\end{abstract}

\section{INTRODUCTION}

The molecular mechanisms that coordinate transmembrane protein trafficking at the cellular level are still unclear. Fluorescence microscopy allows us to localize the distribution of proteins of interest along time. The analysis of the dynamic behavior of these proteins enables to better understand the underlying mechanisms. A common and established approach for extracting this type of information consists in individually tracking particles in fluorescence video-microscopy. The most widespread tracking concept is the correspondence approach [1], [2] which consists in detecting particles independently in each frame and then associating the detected objects over time. The object association is particularly difficult when the density of particles is high, their appearance is similar and their trajectories interact. Kalman filtering [3], particle filtering techniques [4], graph-theory based methods [5] and multiple hypothesis tracking [6] have been developed to improve temporal matching. Practically, these approaches require a high temporal sampling rate to successfully track particles in fluorescence video-microscopy. Unfortunately, the phototoxicity of the acquisition process with this modality limits the number of images that can be acquired. Consequently, a high temporal sampling rate implies a short acquisition time. Another line of work in computer vision considers the object flows between predefined regions in the observed scene to recover the individual object tracks along time [7]. Unfortunately, this method cannot be directly applied to fluorescence microscopy images because of the higher number of objects to track and their possible appearances and disappearances along time while only few occlusions happen in natural scene videos.
The particle flow between cell compartments reveals the relationships between the different cell domains, a generic problem in the study of the cell communication (endocytosis, recycling, motility, etc). Another possible application is the determination of the number of objects interacting with a region of interest over a given period allowing the evaluation of the trigger effect. This approach can also be applied to object flow estimation between non connected regions following the so-called network tomography as described in [8].

In this paper, we describe an original approach to extract global information about trafficking by analyzing the particle flow between connected regions. These regions are arbitrarily defined to form a regular cell tessellation or correspond to a specific cell segmentation such as cell compartments. The flow will be inferred from the variation of the number of particles in each region. In addition to the flow computed between regions in the cell, the number of appearances and disappearances is also estimated by introducing a virtual region. The particle flow estimation proceeds in three steps: i) the cell is partitioned into regions; ii) the particles are detected in the image sequence; iii) the particle flow is estimated at each time step from the particle number variation in each region. Except some trivial cases, flow estimation is an ill-posed problem. Therefore, we investigate two different regularization schemes [9], [10] and compare them to a simple correspondence approach. The particle flow estimation provides less information than a tracking framework as it only describes the global particle paths in the cell compared to individual tracks. However, it offers an efficient way to extract relevant information from the image sequences while further keeping cells alive by reducing the frame rate, which leads to observe biological processes over a longer period of time.

The remainder of the paper is organized as follows. In Section II, we describe our particle flow estimation approach. In particular, we propose two regularizing methods to solve the flow estimation that is an ill-posed problem. In Section III, we compare the performance between flow estimation methods and tracking methods on synthetic and real fluorescence image sequences. Section IV contains concluding remarks. 


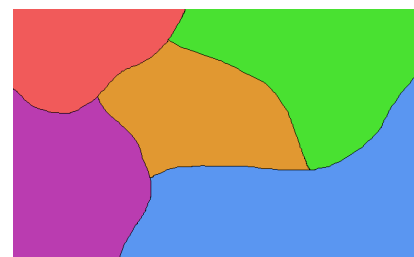

(a)

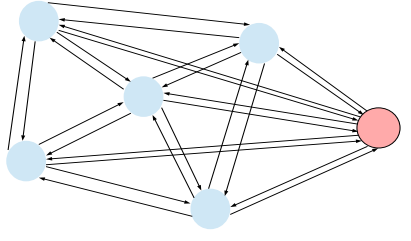

(b)
Fig. 1. (a) Image partition example; (b) graph associated to the image partition (a).

\section{Flow ESTIMATION}

In this section, we introduce the partitioning of the cell and define the associated graph. Then, we present the particle flow estimation approach and we propose two regularizing methods to solve this ill-posed problem.

\section{A. Image partition}

Let us consider a partition of the image domain $\Omega$. An oriented graph $G(E, V)$ with $|E|$ edges and $|V|$ vertices is associated to the partition. The vertices correspond to the regions of the partition and the edges to the boundaries between the regions. A virtual region is added and connected to all the other regions to handle the particles that appear or disappear over time. The graph is oriented since only positive flows between regions are considered. An example of an image partition and the associated graph are shown in Fig.1. In Fig.1(b), the virtual region is represented as a red disk while the other regions appear as blue disks.

Several partition strategies are possible. A very simple partition consists in dividing the image domain into a regular grid of size $n \times m$. In this case, the graph is defined with $|V|=n m+1$ vertices and $|E|=6 n m-2 n-2 m$ edges. If the particle trafficking is known to happen between cell compartments, the segmentation of these compartments supplies an adequate image partition.

\section{B. Flow estimation}

Matching particles is a hard task in fluorescence microscopy, especially when the number of particles is high, appearances and disappearances are frequent and when the frame rate is low. In contrast, we propose to infer the particle flow between regions by evaluating the number of particles in each region at each time step. Let us define $Y(t)=\left\{y_{i}(t), i \in[1,|V|]\right\}$ the number of detected particles in each region $i \in[1,|V|]$ at each time step $t$. The following conservation equation (Kirchhoff law) can be written:

$$
\frac{\partial Y}{\partial t}-M X=0
$$

This equation allows us to associate the particle flow $X(t)=$ $\left\{x_{j}(t), j \in[1,|E|]\right\}$ on each edge $j \in[1,|E|]$ with the temporal variation of the particle number in each region. In this context, the divergence operator $M$ corresponds to a
$|V| \times|E|$ matrix such that for each vertex $i$ and edge $j$ :

$$
m_{i j}=\left\{\begin{aligned}
1 & \text { if edge } j \text { points to vertex } i, \\
-1 & \text { if edge } j \text { originates from vertex } i, \\
0 & \text { otherwise. }
\end{aligned}\right.
$$

As the number of edges is generally higher than the number of vertices, the matrix $M$ has more columns than lines.

The estimation of the flow $X(t)$ from the measures $Z=$ $\partial Y(t) / \partial t$ is performed through the inversion of $M$ or the minimization of $\|Z-M X\|^{2}$. The number of particles that appear or disappear is estimated by introducing the virtual region $i^{*}$ and by computing the total variation of particles between two time steps to be attached to that virtual region:

$$
z_{i^{*}}(t)=-\left(\sum_{i \in V \backslash i^{*}} y_{i}(t+1)-\sum_{i \in V \backslash i^{*}} y_{i}(t)\right) .
$$

As the graph is oriented, the flow on the edges is positive and this constraint can be exploited to constrain the solution. Then, we resort to a least-mean square algorithm with a positivity constraint to estimate the flow if the number of edges is inferior to the number of vertices. As the problem is ill-posed in most cases, we need to regularize the problem. First, we investigate a spectral cut method. More precisely, we estimate the particle flow with the technique proposed by Lawson and Hanson [9], the so-called NNLS algorithm.

The probability that a particle crosses the boundary between two regions is relatively low. Then, we can assume that the flow estimates must be sparse, which leads to another regularization method. As a consequence, we minimize the following global function:

$$
J(X, Z)=\frac{1}{2}\|Z-M X\|^{2}+\kappa|X|+\frac{1}{2} d_{C}^{2}(X),
$$

where $d_{C}$ is the distance to the space $C$ of the positive solutions and $\kappa$ is a real positive parameter. To estimate the flow, we adopt the PPXA algorithm [10] well suited to non differentiable convex minimization problem. It uses the proximal operators associated to the different energy terms involving the proposed constraints (see Algorithm 1). In particular, the operator associated to the square distance function is given by [10]: $\frac{1}{2}\left(x+P_{C}(x)\right)$, where $P_{C}(x)=x$ if $x>0$ and $P_{C}(x)=0$ otherwise.

\section{EXPERIMENTAL RESULTS}

In this section, we compare on synthetic and real data the performance of the two object flow estimation methods described in the previous section along with a nearestneighbor matching (NNM) algorithm.

\section{A. Synthetic data}

Using synthetic data, we aim at evaluating the influence of the number of particles involved, the particle speed, the appearance and disappearance rate, along with the number of regions of the partition. In addition to the two particle flow estimation methods described in Section II, we also consider 


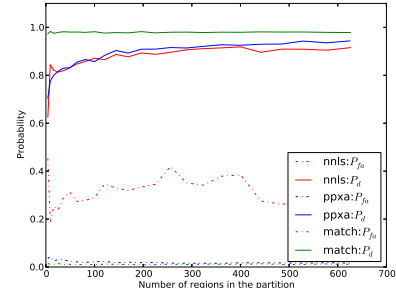

(a)

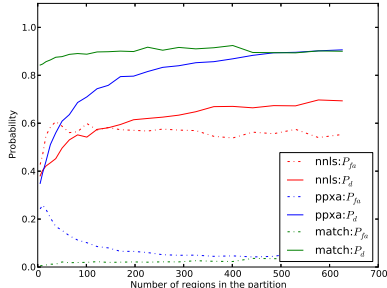

(b)

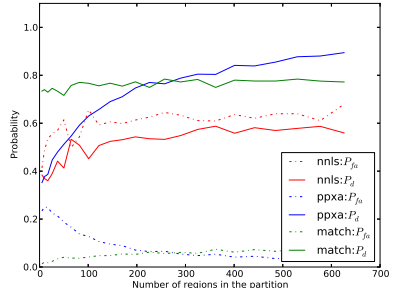

(c)

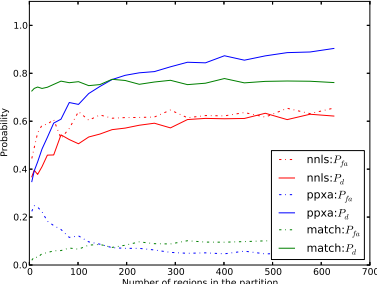

(d)

Fig. 2. Performance evaluation of the NNLS, PPXA and NNM algorithm for four different scenarios. (a-d) Directed motion combined with a random walk, (b-d) object appearances/disappearances, (c-d) object start/stop. The particle speed is equal to 3 pixels per frame in the first three scenarios while it is equal to 10 pixels per frame in the last scenario.

Data: $M, Z, \varepsilon \in[0,1], \gamma>0, \kappa>0$

Result: $X_{N}$

$U_{1,1}=0, U_{2,1}=0$

for $n \in[1, N]$ do

$$
\begin{aligned}
& P_{1, n}=\left(I+\gamma M^{T} M\right)^{-1}\left(U_{1, n}+\gamma M^{T} Z\right) \\
& P_{2, n}=\frac{1}{2}\left(U_{2, n}+P_{C}\left(U_{2, n}\right)\right) \\
& P_{3, n}=\max \left(U_{3, n}-\frac{1}{\kappa}, 0\right) \operatorname{sign}\left(U_{3, n}\right) \\
& P_{n}=P_{1, n}+P_{2, n}+P_{3, n} \\
& \varepsilon \leq \lambda_{n} \leq 2-\varepsilon \\
& \text { for } i \in[1,3] \mathbf{d o} \\
& \quad U_{i, n+1}=U_{i, n}+\lambda_{n}\left(2 P_{n}-X_{n}-P_{i, n}\right) \\
& \text { end } \\
& X_{n+1}=X_{n}+\lambda_{n}\left(P_{n}-X_{n}\right)
\end{aligned}
$$

end

Algorithm 1: PPXA minimization algorithm used to estimate the particle flow with proximal operators associated to the different constraints.

a simple Nearest-Neighbor Matching (NNM) algorithm. The latter consists in pairing each particle at time $t$ to the nearest particle at time $t+1$. The particles that cross a boundary between two regions in the partition contribute to the flow. The particle flow estimated for each method is compared to the ground truth. For each simulation, the true positive probability (sensibility) $P_{d}$ is defined as the ratio between the number of correct detections and the total number of particles that cross a boundary between two regions over the sequence. The probability of false alarm (specificity) $P_{f a}$ is defined as the ratio between the number of false alarms and the number of boundary crosses.

Four different scenarios with 50 particles are simulated. All the scenarios correspond to a directed motion combined with a random walk. In the second scenario, the particle appear and disappear with a probability equal to 0.1 . In the two last scenarios, on top of appearances and disappearances, the particles can stop at a given location with a probability equal to 0.8 , and start over with a probability equal to 0.2 . In the three first scenarios, the particle speed is equal to 3 pixels per frame while it is equal to 10 pixels per frame in the last scenario. The number of regions in each simulation varies in the range $[4,625]$. For each setting, we report the average result over 10 simulations for each of the three methods. To supply a ground truth, we compute the crossing of the

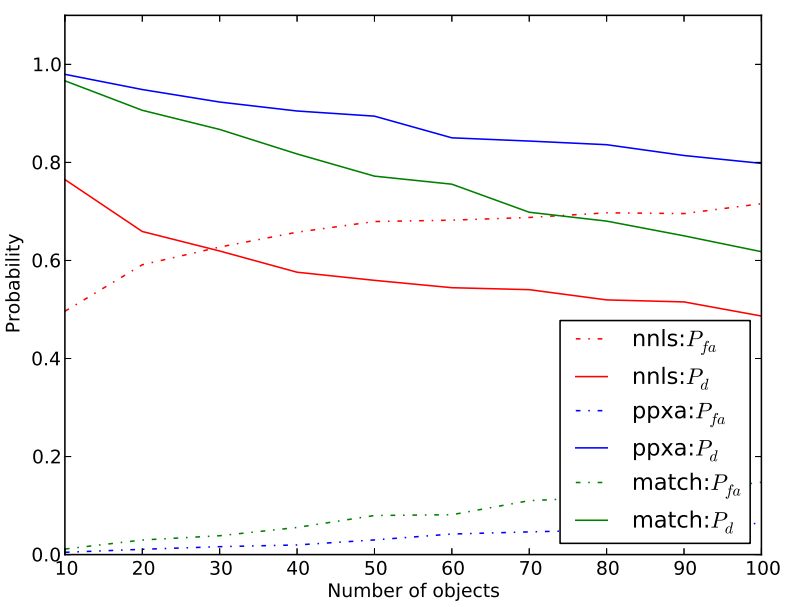

Fig. 3. Evaluation of the influence of the number of objects for a directed motion combined with a random walk, with particle appearances/disappearances and start/stop.

simulated trajectories with the boundaries of the regions. The time computation for the NNM algorithm increases as $K^{2}$. The time computation for the NNLS and the PPXA algorithms does not depend on the number $K$ of particles but on the number of edges in the graph.

As shown in Fig. 2, the NNM algorithm is almost insensitive to the image partition, except for the $P_{f a}$ value that increases slowly with the number of regions. But the $P_{d}$ value decreases when vesicles can appear/disappear and start/stop. This value also decreases a little when the speed increases. Except in the first scenario, the PPXA algorithm shows higher $P_{d}$ values and lower $P_{f a}$ values than the NNM algorithm. The particle appearances/disappearances and start/stop necessitate to consider a higher number of regions to obtain satisfying results. The NNLS algorithm shows a similar behaviour than the PPXA algorithm but the $P_{d}$ values (resp. $P_{f a}$ values) are lower (resp. higher). This demonstrates the importance of the sparsity constraint on the particle flows.

The influence of the number of particles on the results is evaluated and illustrated in Fig. 3 for the scenario shown in Fig. 2 (c). These results demonstrate the higher $P_{d}$ values and lower $P_{f a}$ values obtained with the PPXA algorithm when compared to the NNM and NNLS algorithms whatever the 


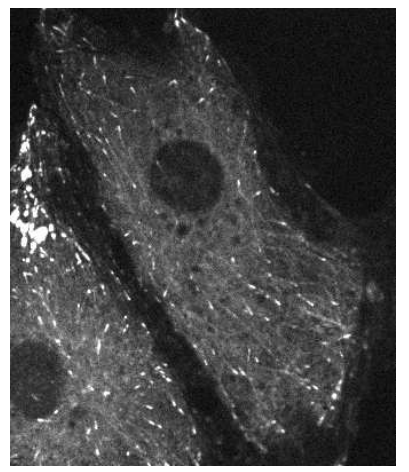

(a)

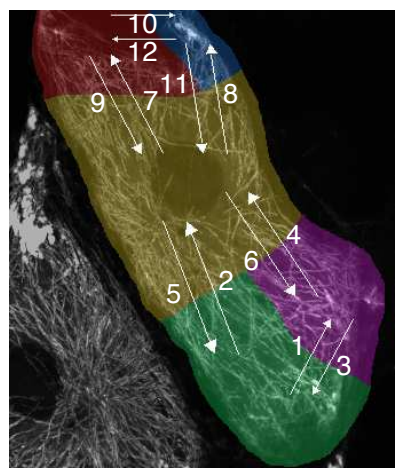

(b)
Fig. 4. (a) Image \#1 of the sequence; (b) edge numbers between the regions.

\begin{tabular}{|c|c|c|c|c|c|c|c|}
\hline $\begin{array}{c}\text { Edge } \\
\text { number }\end{array}$ & $\begin{array}{c}\text { Real } \\
\text { flow }\end{array}$ & $\begin{array}{c}\text { NNM } \\
\text { every } \\
\text { frames }\end{array}$ & $\begin{array}{c}\text { NNM } \\
\text { every } \\
\text { frames }\end{array}$ & $\begin{array}{c}\text { NNM } \\
\text { every } \\
5 \text { frames }\end{array}$ & $\begin{array}{c}\text { PPXA } \\
\text { every } \\
\text { frames }\end{array}$ & $\begin{array}{c}\text { PPXA } \\
\text { every } \\
\text { 2frames }\end{array}$ & $\begin{array}{c}\text { PPXA } \\
\text { every } \\
5 \text { frames }\end{array}$ \\
\hline 1 & 13 & 16 & 15 & 13 & 16 & 16 & 14 \\
\hline 2 & 0 & 4 & 4 & 5 & 6 & 7 & 5 \\
\hline 3 & 5 & 15 & 15 & 9 & 8 & 6 & 8 \\
\hline 4 & 3 & 1 & 3 & 2 & 4 & 5 & 4 \\
\hline 5 & 23 & 24 & 26 & 13 & 22 & 20 & 14 \\
\hline 6 & 9 & 10 & 13 & 6 & 10 & 10 & 4 \\
\hline 7 & 14 & 19 & 14 & 8 & 16 & 16 & 11 \\
\hline 8 & 12 & 10 & 14 & 5 & 14 & 12 & 9 \\
\hline 9 & 2 & 4 & 4 & 2 & 5 & 5 & 4 \\
\hline 10 & 3 & 7 & 7 & 3 & 10 & 13 & 2 \\
\hline 11 & 0 & 2 & 2 & 2 & 3 & 1 & 3 \\
\hline 12 & 1 & 3 & 5 & 3 & 5 & 5 & 2 \\
\hline \hline MAE & - & 3.16 & 3.08 & 3.33 & 3 & 3.08 & 3.08 \\
\hline
\end{tabular}

\section{TABLE I}

REAL AND ESTIMATED VESICLE FLOWS WITH THE NNM AND PPXA ALGORITHMS WHEN CONSIDERING ALL THE FRAMES IN THE SEQUENCE, EVERY TWO FRAMES AND EVERY FIVE FRAMES. THE MEAN ABSOlute

ERROR (MAE) IS REPORTED IN THE LAST ROW FOR THE TWO ALGORITHMS AND THE THREE DIFFERENT SAMPLING RATES.

number of particles is.

\section{B. Real TIRF image sequence}

We compare the PPXA algorithm and the NNM algorithm on a real image sequence (Fig. 4(a)) of 237 frames of size $337 \times 400$ acquired in TIRF microscopy and showing the protein clip170 fluorescently labeled. This sequence is particularly challenging because of the high number of vesicles with varying speed. Furthermore, many vesicle appearances and disappearances happen as the acquired volume is thin in TIRF microscopy. The ground truth is defined by visually inspecting the image sequence. The results obtained with the NNM and PPXA algorithms for different sampling rates are reported in table I. We consider a simple partition of 5 regions (see Fig. 4(b)) to have a global idea about vesicle trafficking over the sequence. As the PPXA algorithm needs a high number of regions to obtain satisfying results, a partition of 730 regions is used and the final results are converted and reported in the partition with 5 regions. These results indicate that the PPXA algorithm better estimates the vesicle flows between the regions.

\section{CONCLUSiON}

We have proposed an original approach that determines the flow of particles between regions issued from a partition of the image domain. In contrast to usual approaches, we do not track individual particles, we only need to locally count particles on regions over time and minimize a global energy function. We have specified three methods to determine the particle flow. We have conducted comparative experiments on synthetic and real fluorescence image sequences. We have shown that adding a sparsity constraint on the number of detected events allows us to reduce the number of false alarms. Compared to usual tracking methods, our approach is simpler and the results are very stable with respect to the only two parameters. We have thus demonstrated the efficiency and the contributions of this approach.

\section{ACKNOWLEDGMENTS}

The authors would like to thank Hélène de Forges and Franck Perez (Institut Curie, Paris) for providing the data set. They also acknowledge France-BioImaging infrastructure supported by the French National Research Agency (ANR10-INBS-04-07, "Investments for the future").

\section{REFERENCES}

[1] M. Cheezum, W. Walker, and W. Guilford, "Quantitative comparison of algorithms for tracking single fluorescent particles," Biophysical Journal, vol. 81, no. 4, pp. 2378-2388, 2001.

[2] I. Sbalzarini and P. Koumoutsakos, "Feature point tracking and trajectory analysis for video imaging in cell biology," Journal of Structural Biology, vol. 151, no. 2, pp. 182-195, 2005.

[3] A. Genovesio, T. Liedl, V. Emiliani, W. Parak, M. Coppey-Moisan, and J. Olivo-Marin, "Multiple particle tracking in 3-d+t microscopy: method and application to the tracking of endocytosed quantum dots," IEEE Transactions on Image Processing, vol. 15, no. 5, pp. 10621070, 2006.

[4] I. Smal, E. Meijering, K. Draegestein, N. Galjart, I. Grigoriev, A. Akhmanova, M. E. van Royen, A. B. Houtsmuller, and N. Niessen, "Multiple object tracking in molecular bioimaging by RaoBlackwellized marginal particle filtering," Medical Image Analysis, vol. 12 , no. 6 , pp. 764-777, 2008.

[5] K. Jaqaman, D. Loerke, M. Mettlen, H. Kuwata, S. Grinstein, S. Schmid, and G. Danuser, "Robust single-particle tracking in live-cell time-lapse sequences," Nature Methods, vol. 5, pp. 695-702, 2008.

[6] N. Chenouard, I. Bloch, and J.-C. Olivo-Marin, "Multiple hypothesis tracking in microscopy images," in IEEE Int. Symp. on Biomedical Imaging: from nano to macro (ISBI'09), Boston, MA, July 2009, pp. 1346-1349.

[7] J. Berclaz, F. Fleuret, E. Turetken, and P. Fua, "Multiple objects tracking using K-shortest paths optimization," Pattern Analysis and Machine Intelligence, vol. 33, no. 9, pp. 1806-1819, 2011.

[8] T. Pécot, C. Kervrann, and P. Bouthemy, "Minimal paths and probabilistic models for origin-destination traffic estimation in live cell imaging," in IEEE Int. Symp. on Biomedical Imaging: from nano to macro (ISBI'08), May 2008, pp. 843-846.

[9] C. Lawson and R. Hanson, Solving Least Squares Problems. Society for Industrial and Applied Mathematics, 1974.

[10] P. L. Combettes and J.-C. Pesquet, Fixed-Point Algorithms for Inverse Problems in Science and Engineering. Springer, New York, 2011, ch. 10. Proximal splitting methods in signal processing, pp. 185-212. 